\title{
The Progenitor Stars of Core-Collapse Supernovae
}

\author{
Stephen J. Smartt ${ }^{1}$, R. Mark Crockett ${ }^{1}$, John J. Eldridge ${ }^{2}$ \\ and Justyn R. Maund ${ }^{3}$ \\ ${ }^{1}$ Astrophysics Research Centre, School of Mathematics and Physics, \\ Queen's University Belfast, Belfast BT7 1NN \\ ${ }^{2}$ Institute of Astronomy, The Observatories, University of Cambridge, \\ Madingley Road, Cambridge CB3 \\ ${ }^{3}$ Department of Astronomy and McDonald Observatory, University of Texas, \\ 1 University Station C1402, Austin, TX 78712-0259
}

\begin{abstract}
Knowledge of the nature and mass of the progenitor stars of core-collapse supernovae are critical elements to test theoretical models of stellar evolution and stellar explosions. Here we describe the current limits and restrictions that can be placed on the progenitor stars of type II SNe and those of Ib/c. There are detections of some type II-P SN progenitors but the exploding stars that produce type $\mathrm{Ib} / \mathrm{c}$ have eluded discovery. We discuss implications of these quantitative limits and the conclusions that we can now draw.
\end{abstract}

Keywords. supernovae: general - stars: evolution - supergiants

\section{Introduction}

There is huge diversity in the spectral and photometric evolution of supernovae (SNe) that arise from the death of massive stars. The diversity is much more pronounced than for the thermonuclear type Ia SNe. As discussed at this conference (see the contributions from Burrows, Dessart et al) the long favoured paradigm for energy release is the collapse of an iron core at the end of the nuclear burning life of a massive star. The observed core-collapse SN (CCSN) types are then thought to be related to the state of the stellar envelope when this explosion occurs deep in the heart of the star. The mass range that will produce CCSNe and the exact relation between CCSN type and progenitor star is now an area in which observers can contribute valuable and fundamental information. The core-collapse paradigm has even been challenged recently (for example, see Nathan Smith's contribution to this volume) with the exotic pair-instability or pair-creation process suggested to be responsible for some extremely luminous SNe. The process that produces gamma-ray bursts in some way also creates SNe (of types Ic and Ib) $\dagger$ hence it is important that we understand the nature of stars before they collapse to allow theoretical models of stellar structure and the core-collapse mechanism to be studied. With the availability of easy access HST and large ground-based archives searching for progenitor stars has become almost routine for nearby events. As will be shown in this paper, although the search mechanism is rather straightforward the detection of massive progenitors has not been easy. In fact it is somewhat surprising how few definite detections have been

$\dagger$ At the time of the conference in Dec. 2007 only type Ic SNe had been associated with GRBs or X-ray flashes. However in January 2008 a SN Ib (SN2008D) was found to be preceded by an Swift discovered XRF. 


\section{The relative rates of the supernovae types}

In this paper we are presenting a summary of the results for the search for CCSN progenitors over a 10 year period. We have tended to apply a distance limit of $\sim 25-30 \mathrm{Mpc}$ when searching for SNe which are close enough that the massive stellar populations (at least the brightest stars) are resolved and detectable. Hence to make this search meaningfully complete and remove as much bias as possible we restrict this survey to galaxies within $V_{\text {vir }}<2000 \mathrm{~km} \mathrm{~s}^{-1}$, which corresponds to a distance of around $27 \mathrm{Mpc}$. It is interesting to also study the rates within this volume, as one would presume this reflects the initial mass function (IMF), stellar evolution, and in some way the influence of binarity. We have checked every SN reported to the IAU and found all those in galaxies which, after correction for the infall of the Local Group towards the Virgo cluster, lie within the velocity range. We have compiled the types from the most up to date literature sources, unpublished but publicly available spectra and photometry (including some amateur lightcurves) and assigned a most probable type. Out of the $135 \mathrm{SNe}$ that make it into our catalogue only 2 have not been spectroscopically classified. The breakdown of the types is listed in Table 1. It is interesting to note that only one of these SNe has been reported to have shown spectral and photometric evolution that is similar to SN1987A : that is 1998a (Pastorello et al. 1995). Hence the rate of the explosions of blue supergiants does not appear to be large. At the time SN1987A was found, it was suggested by several authors that the faint magnitude during its early evolution $\left(M_{V} \sim-16\right)$ could mean many SNe of this type have gone undiscovered (see for example Arnett et al. 1989). The last 10 years of SNe discoveries show that is unlikely and they are intrinsically rather rare.

\section{Detection of progenitor stars}

Apart from the now well known cases of SN1987A and SN1993J there are relatively few cases were a progenitor star has been identified. Our group, together with others around the globe (e.g. the UC Berkeley, Caltech and STScI groups) have been searching hard for the highest quality images of nearby galaxies in well maintained archives that allow interesting limits to be set on the nature of progenitor stars Although there were some attempts to do this with the early HST archive (e.g. Barth et al. 1996) it was not until the late 1990's that the HST archive became rich enough to allow the systematic searching for progenitor stars. Since around 1999, with the publication of interesting limits on the two very close SNe 1999em and 1999gi (Smartt et al. 2001, Smartt et al. 2002) there have been frantic attempts to identify progenitors soon after the explosion of nearby supernovae (e.g. Smartt et al. 2004, Li et al. 2006, Gal-Yam et al. 2005, Maíz-Apellániz et al. 2004). Astronomers interested in this field should beware, there are often many claims and counter-claims in the early days after an explosion in which the astrometric positioning of the supernova on the pre-explosion image (using ground-based astrometry) often leads to wrong results (e.g. Smartt et al. 2001, Richmond \& Modjaz 2005, Li et al. 2004).

The most valuable source of archive imaging is of course HST due to its exquisite resolution across the optical and NIR, the well calibrated and easy to search archive, and the depth it achieves in exposures of even a few hundred seconds. However this resolution requires that the follow-up image that is used to astrometrically match the images is of similar resolution. Average seeing ground-based images are normally not good enough to unambiguously identify progenitor stars in HST images. The uncertainty in the alignment is usually of the order $\sim 70-100$ milliarcseconds, and often this is not 
Table 1. The relative frequency of SNe types within a fixed volume and in a fixed time period of 10 years.

\begin{tabular}{rlll}
\hline & & Relative Rates & Core-Collapse Only Rates \\
\hline Type & No. & (per cent) & (per cent) \\
\hline II-P & 53 & 39.3 & 59.6 \\
II-L & 2.5 & 1.9 & 2.8 \\
IIn & 3.5 & 2.6 & 3.9 \\
IIb & 5 & 3.7 & 5.6 \\
Ib & 8 & 5.9 & 9.0 \\
Ic & 17 & 12.6 & 19.1 \\
Ia & 37 & 27.4 & $\ldots$ \\
LBVs & 7 & 5.2 & $\ldots$ \\
Unclassified & 2 & 1.5 & $\ldots$ \\
\hline Total & 135 & 100 & 100 \\
Total CCSNe & 89 & 66 & 100 \\
\hline
\end{tabular}

good enough to definitively associate a progenitor star with a SN (Crockett et al. 2007). Follow-up HST images or adaptive-optics assisted ground-based images are essential. An example is shown in Fig 1. In this case the astrometric accuracy of the alignment between the HST pre-explosion and the Gemini AO image is 20 milliarcseconds (Crockett et al. 2008).
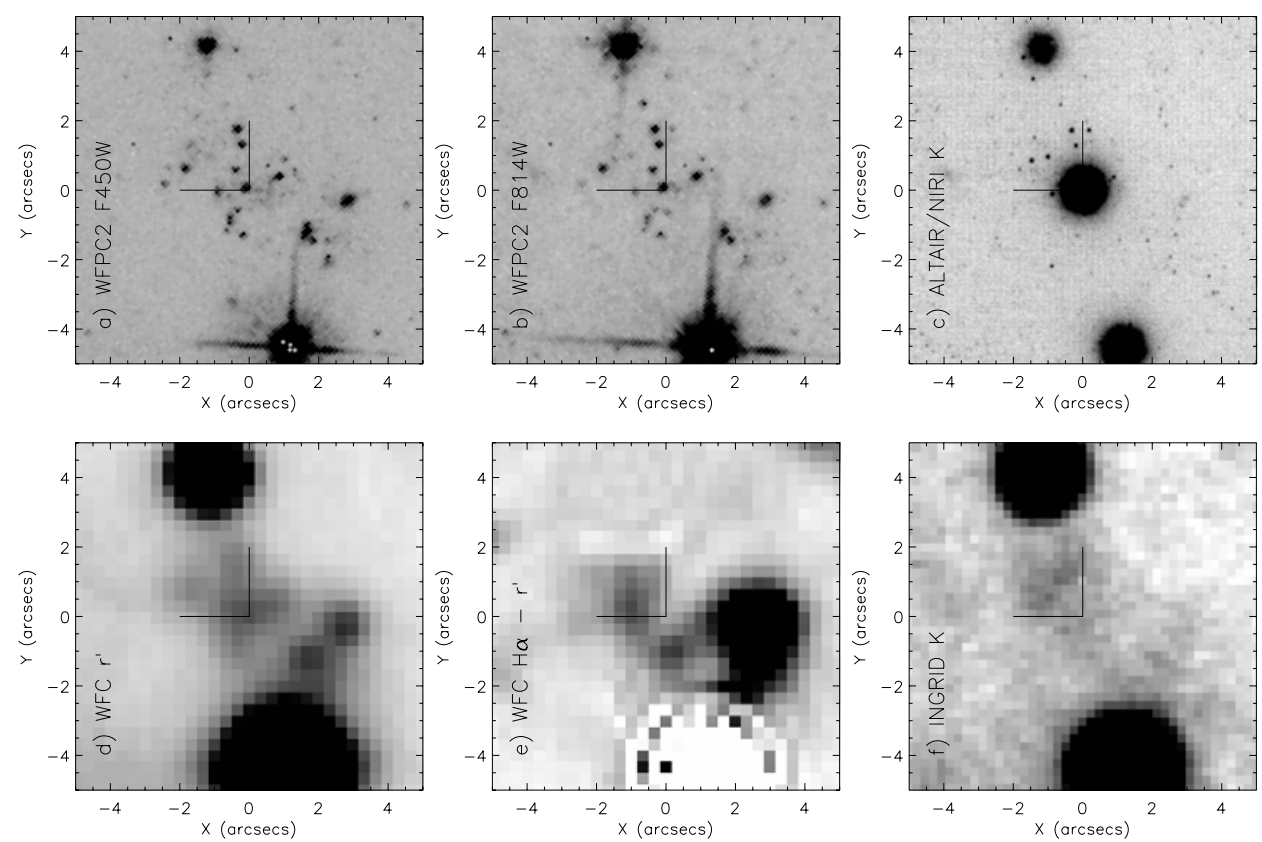

Figure 1. Figure taken from Crockett et al. (2008) which studied the pre-explosion environment of the type Ic SN2007gr. This series of images nicely illustrates the resolution of typical archive imaging from HST, ground-based imaging in average conditions (around 1 arcsecond quality) and diffraction limited $K$-band imaging from Gemini North and the Altair AO system. In this case the SN was shown to be very close, but not exactly coincident with, a bright point source of absolute magnitude of $M_{\mathrm{F} 450 \mathrm{~W}}=-9.0$. Crockett et al. (2008) suggest this may be a compact star cluster. 


\section{The Progenitor stars of Type II-P SNe}

Smartt et al. (2008) will present a full list of all the nineteen Type II SNe which are within the volume limit as discussed above and which have deep enough pre-explosion images that they set interesting and restrictive limits on the progenitor stars. We have reanalyzed these with a consistent approach, homogeneous treatment of errors and application of one set of stellar evolutionary models. This removes systematic uncertainties when one compares results from the various published mass limits in the literature. The models we use are the Cambridge STARS tracks (Eldridge \& Tout 2004). Seventeen of them are confirmed type II-P SNe from their lightcurves and spectra (the other two being of type II, but lack of photometric monitoring prevents a definitive subtype). Three of these have red supergiant progenitors detected (2003gd, 2004A and 2005cs) all with masses in the range $8_{-2}^{+5} \mathrm{M}_{\odot}$, one is of unknown colour and its magnitude implies a likely mass of $16_{-4}^{+6} \mathrm{M}_{\odot}(1999 \mathrm{ev})$ and two are in compact stellar clusters with turn-off masses around 10-15M $\odot$ (2004am and 2004dj ; Maíz-Apellániz et al. 2004 and Matilla et al. 2008). The rest all have upper limits as for example derived in Smartt et al. (2001, 2002). These upper limits assume that the progenitors were red supergiants at the time of their explosion, as one needs to assume a bolometric correction to apply to the observed magnitude limit to estimate a bolometric luminosity limit and hence a mass. This assumption is probably justified as the vast majority of the sample (17 out of 19) are confirmed II-P, and have fairly similar characteristics in terms of plateau lengths (Arnett 1980).

With these measurements of progenitor masses and upper limits it is now possible to estimate the parameters that describe the progenitor population. The three parameters that we are interested in are the minimum initial mass for a type IIP SN, the maximum initial mass and the IMF of the progenitors. We have employed an unbinned maximum likelihood method (e.g. as employed in Jegerlehner et al. 1996) and calculated the most likely values of minimum mass and upper mass that will produce the mass distribution that we see, assuming that the slope of the IMF is Salpeter $(\alpha=-1.35)$. There appears to be no strong evidence in the Local Group that the IMF significantly variest. The results can be seen in Figure 2. The parameters we estimate are therefore $m_{\min }=7.5_{-1.5}^{+1} \mathrm{M}_{\odot}$ and $m_{\max }=15_{-2}^{+3} \mathrm{M}_{\odot}$. We have also compared the mass - final luminosity relation of other models and find no major differences between those and the STARS code we employ here.

The lower mass limit is consistent with recent estimates for the upper mass limit that will produce a white dwarf. Dobbie et al.(2006) and Williams (2007) suggest that stars up to $6.8-8.6 \mathrm{M}_{\odot}$ may produce white dwarfs. The upper mass limit however is problematic. We see red supergiants in the LMC and SMC with masses up to $20-25 \mathrm{M}_{\odot}$ (see the contributions by Massey \& Levesque at this conference), but why do we not see such high mass SN progenitors? The metallicity of the galaxies at the positions of the SNe are in the LMC-Solar range, thus one would not expect that all stars above $\sim 18 \mathrm{M}_{\odot}$ would enter the WR phase. These more massive red supergiants would be rather easy to detect in the available pre-explosion images. We estimate that if the progenitors are from a massive stellar population with an Salpeter IMF, we would be missing around 3 massive progenitors in this sample. This is not a clear cut result yet, but is something that we will have to worry about if we continue not to detect massive progenitors.

$\dagger$ Outside perhaps the Galactic Center environment, see Don Figer's review, this volume 


\section{The Progenitor stars of Type Ib/Ic SNe}

There have been no detections of a normal quiescent star at the position of any nearby type Ib/c SN despite around 10 attempts. The intriguing case of SN2006jc is the only event where one can claim that there was an object detected before explosion. In this case it appears that an LBV-like outburst was coincident with the SN, two years before explosion (Pastorello et al. 2007) The SN appeared to be Ic-like but enshrouded in a He rich envelope, which was likely ejected in the pre-collapse outburst, which has led to a "Ib-n" suggested classification.

Apart from this there is no detection of the suspected massive WR stars at the position of any Ib/c, and the deepest limits come from SN2002ap (Crockett et al. 2007) which has a pre-explosion detection limit of $M_{B} \simeq-4.3$. Other detection limits are in the absolute magnitude range (in $B, V, R$ like filters) of -5 to -7 . These limits would be sensitive to a large fraction of the typical WR population of the Magellanic Clouds, the Milky Way and M31 (see discussion in Crockett et al. 2007). However WR stars have a wide range in optical magnitudes due to their high and wide ranging temperatures of between -2 to -7 . We are currently working to interpret these observational limits in terms of the WR populations we see locally. A very simple test we can do to check if WR stars are viable progenitors of all the $\mathrm{Ib} / \mathrm{c} \mathrm{SNe}$ is as follows. We hypothesize that the WR LMC population is indeed the sole progenitor population of type Ib/c SNe. Then we can estimate the probability that all of 6 events (for which we have deep pre-explosion

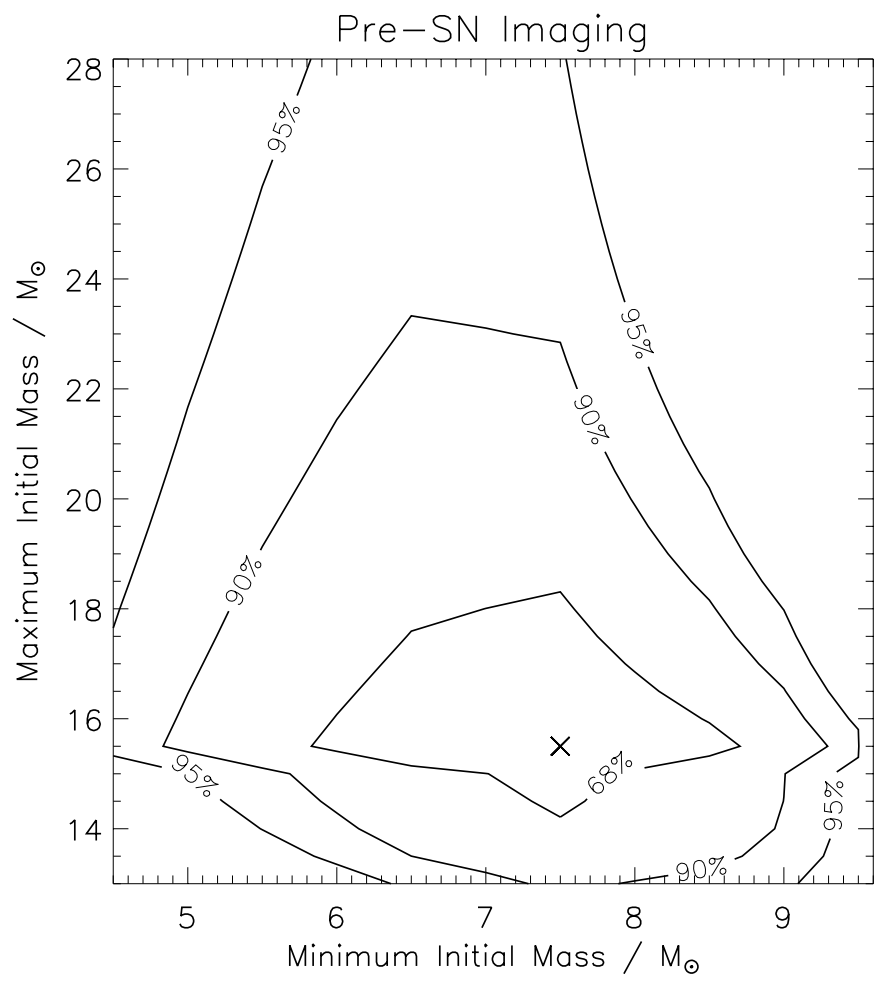

Figure 2. Figure taken from Smartt et al. (2008). This shows the likelihood contours for the minimum and maximum masses for type II-P SNe from the pre-explosion masses compiled from the literature and carefully re-analysed with one method and one stellar evolution code. This suggests a quite low mass for the minimum initial mass that can support a SN explosion. 
limits) have not been detected due to statistical chance. This is simply the product of the individual probabilities and we estimate around 10 per cent. We still have to add in 4 other non-detections. Hence this suggests to us that the initial hypothesis is false, and that there is a fairly substantial population of progenitors that are not from initially very massive stars. These could be lower mass stars in interacting binaries, although there are not large numbers of these systems known in the Galaxy.

In summary then we have derived a most likely mass range for type II-P progenitors which includes a lower mass limit to produce a supernova explosion of around $m_{\min }=$ $7.5_{-1.5}^{+1} \mathrm{M}_{\odot}$. The best estimate of the upper mass limit is $m_{\max }=15_{-2}^{+3} \mathrm{M}_{\odot}$. This is not consistent with the high mass red supergiant population in the Magellanic Clouds and it remains to be seen why this discrepancy exists. We postulate that higher mass stars may form black holes and faint explosions which are perhaps going undetected in current $\mathrm{SNe}$ surveys. Finding such a population would be a stunning discovery. It also appears that the observed Ib/c SN population cannot all be explained by massive Wolf-Rayet stars. Both the high rate of local $\mathrm{Ib} / \mathrm{c}$ SNe and the lack of detections of any progenitors support this. We suggest that this is strong evidence that there must be an additional population of $\mathrm{He}$ stars and stripped CO stars within interacting binaries (e.g. see the models of Nomoto et al. 1995). Such a large population of progenitors have so far remained undiscovered in the Milky Way and the Local Group.

\section{References}

Arnett, W. D. 1980, ApJS, 237, 541

Arnett, W. D., Bahcall, J. N., Kirshner, R. P., \& Woosley, S. E. 1989, ARA\& A, 27, 629

Barth, A. J., van Dyk, S. D., Filippenko, A. V., et al. 1996, AJ, 111, 2047

Crockett, R. M., Smartt, S. J., Eldridge, J. J., et al. 2007, MNRAS, 381, 835

Crockett, R. M., Maund, J. R., Smartt, S. J., et al. 2008, ApJ, 672, L99

Dobbie, P. D., Napiwotzki, R., Burleigh, M. R., et al. 2006, MNRAS, 369, 383

Eldridge, J. J. \& Tout, C. A. 2004, MNRAS, 353, 87

Gal-Yam, A., Fox, D. B., Kulkarni, S. R., et al. 2005, ApJ, 630, L29

Jegerlehner, B., Neubig, F., \& Raffelt, G. 1996, PRD, 54, 1194

Li, W., Filippenko, A. V., \& van Dyk, S. D. 2004, IAUC, 8388, 2

Li, W., Van Dyk, S. D., Filippenko, A. V., et al. 2006, ApJ, 641, 1060

Maíz-Apellániz, J., Bond, H. E., Siegel, M. H., et al. 2004, ApJ, 615, L113

Mattila, S., Smartt, S. J., et al. 2008, MNRAS, in prep.

Nomoto, K. I., Iwamoto, K., \& Suzuki, T. 1995, Phys. Rep., 256, 173

Pastorello, A., Baron, E., Branch, D., et al. 2005, MNRAS, 360, 950

Pastorello, A., Smartt, S. J., Mattila, S., et al. 2007, Nature, 447, 829

Richmond, M. W. \& Modjaz, M. 2005, IAUC, 8555, 2

Smartt, S. J., Gilmore, G. F., Trentham, N., et al. 2001, ApJ, 556, L29

Smartt, S. J., Gilmore, G. F., Tout, C. A., \& Hodgkin, S. T. 2002, ApJ, 565, 10

Smartt, S., Ramirez-Ruiz, E., \& Vreeswijk, P. 2002, IAUC, 7816, 3

Smartt, S. J., Maund, J. R., Hendry, M. A., et al. 2004, Science, 303, 499

Smartt, S. J., et al. 2008, MNRAS, in prep.

Williams, K. A. 2007, in: R. Napiwotzki \& M. R. Burleigh (eds.), 15th European Workshop on White Dwarfs (San Francisco: ASP), ASP Conf. Ser., 372, 85

\section{Discussion}

N. Smith: I'm wondering about events like 1987A, the SN started out faint and the progenitor was a compact star. Are such SNe and progenitors under-represented because of selection effects or are they intrinsically rare? 
SMARTT: I think they are intrinsically rare. 87A was not so faint, with a peak during its plateau of -16 , so such events would be detectable by the various surveys that have produced the 100 nearby SNe in the last $10 \mathrm{yrs}$. Only one of these events has a lightcurve and optical spectrum that one would confidently identify as 87A-like, so I think they are very rare. The progenitor itself was quite massive, around 20 solar masses, and such B-type supergiants would be detectable in many of the archive images. So again I don't think we are biassed against them.

CROWTHER: It seems clear that not all type Ib/c SNe result from very massive WN or WC stars since type Ic appear more common than type Ib SN (yet WN are more common than WC stars), plus these SN are often not associated with massive star forming regions in their host galaxies.

SMARTT: Yes, I agree but if all stars above 20 solar masses do actually explode one might expect that some fraction of our sample do include such massive stars.

LEITHERER: What limits your success rate, is it depth of the image or crowding of the field? A related question: could you miss some SN progenitors if they are heavily reddended or in a crowded field?

SMARTT: Surprisingly few SNe in my list were in very crowded regions, and only two are coincident with compact, spatially unresolved clusters (2004dj and 2004am). The major factor limiting the success rate of detecting progenitors is the depth of the archive images. I think we probably are missing some nearby SNe which are reddened by more than 3 mags in the visual, there are very few of these found and those that are tend to be very close $(3-6 \mathrm{Mpc})$. It might be that the more massive progenitors produce these type of events, which are missing because the SNe are not discovered.

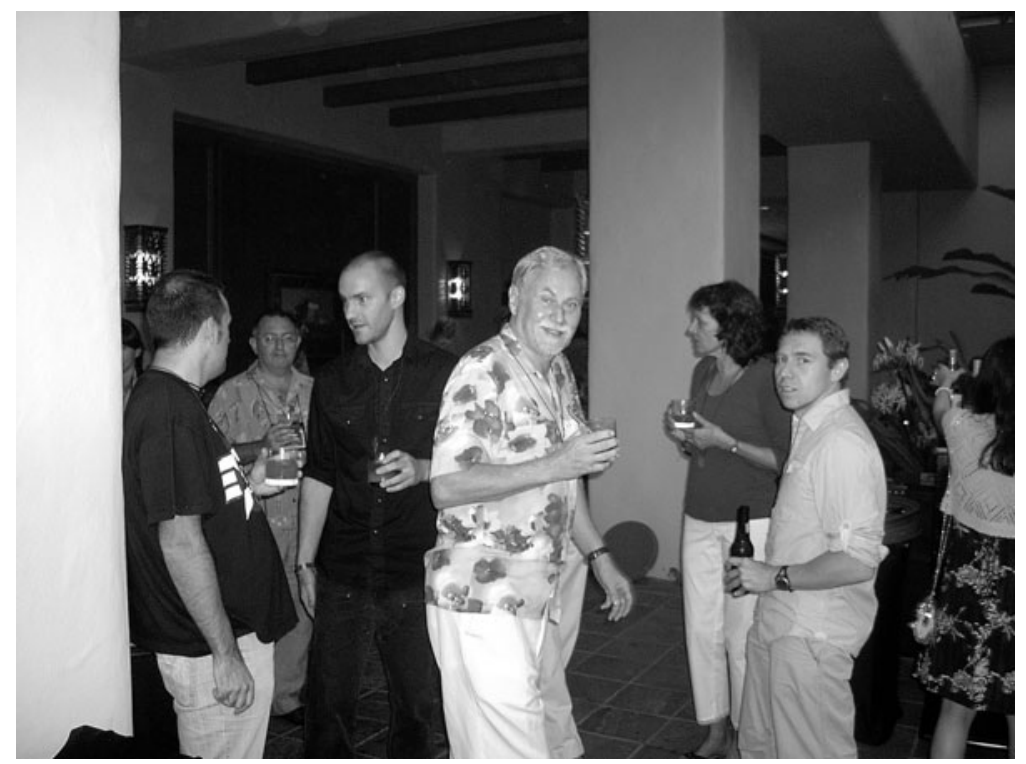

Nathan Smith (center left), Rolf Kudritzki (center right) and Steve Smartt (right). 


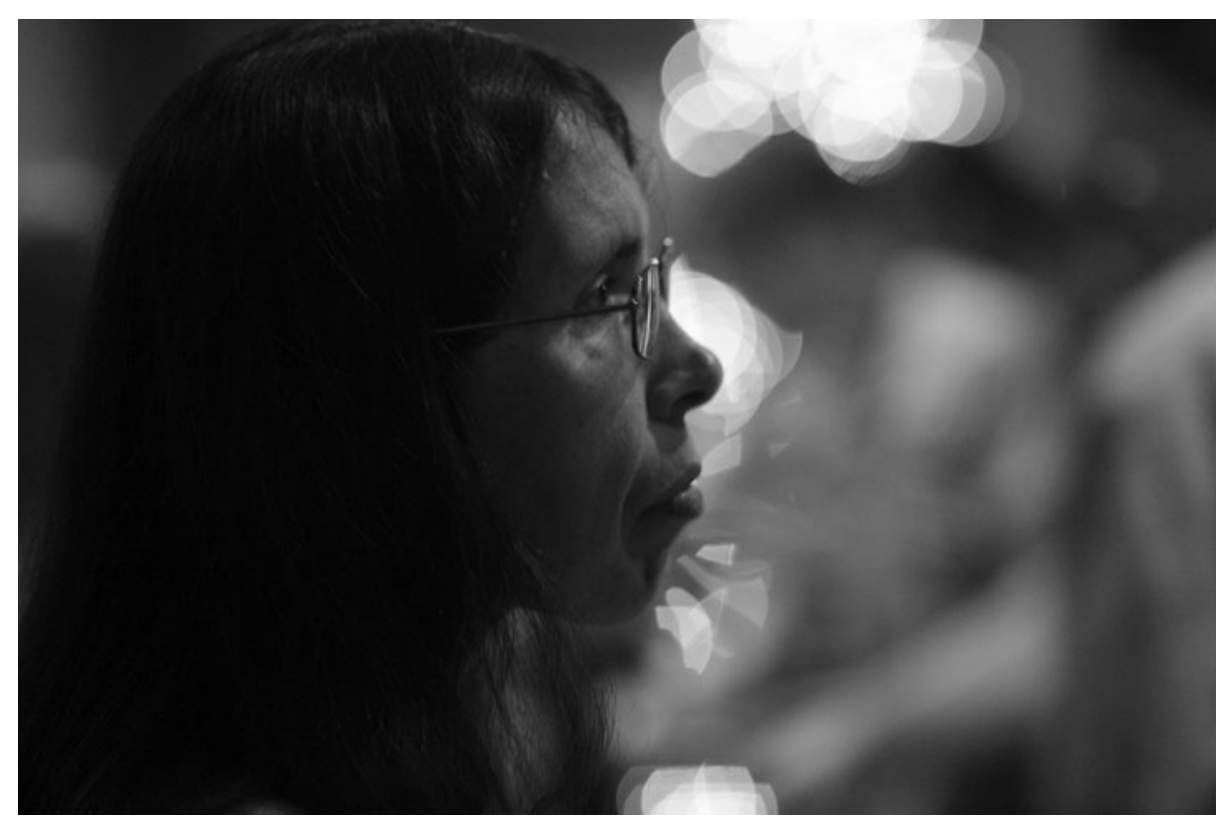

Margarita Rosado.

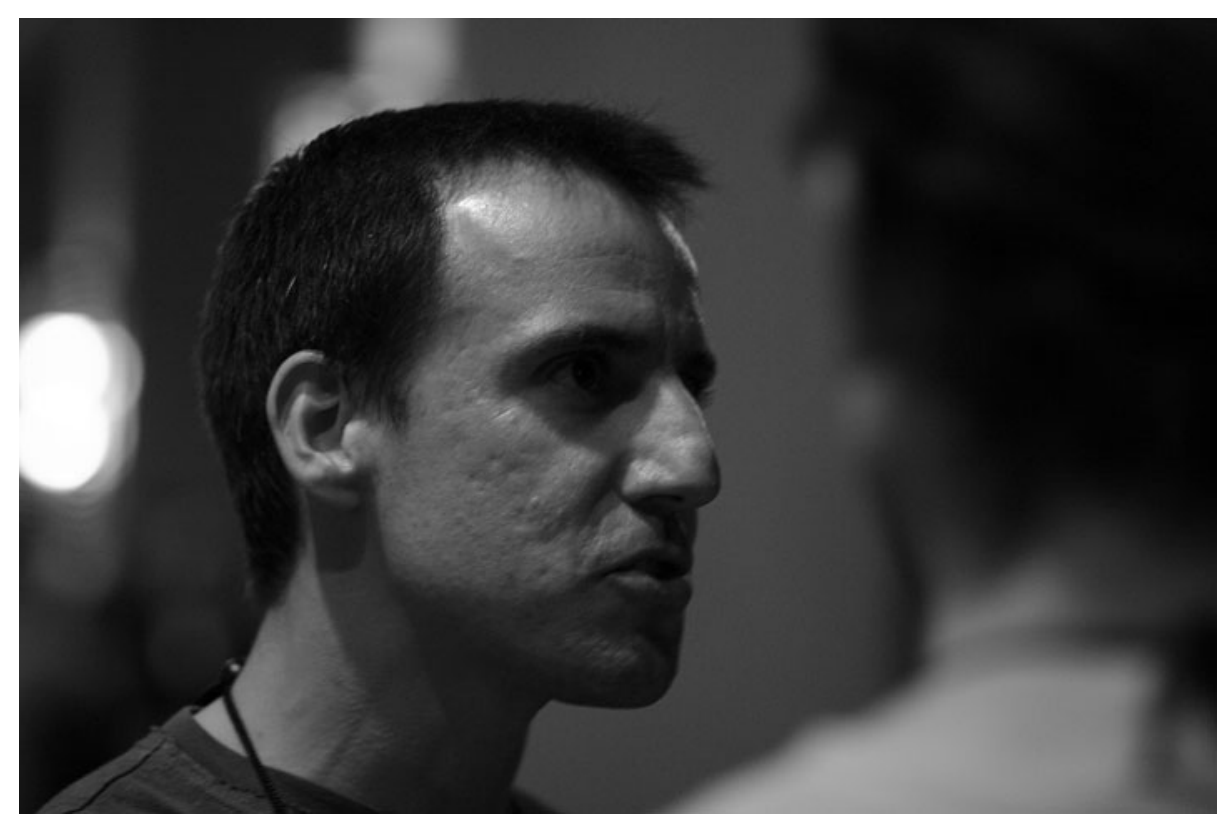

Jean-Claude Bouret. 